\title{
Progress in Research of Oral Health in Patients with Type 2 Diabetes Quality of Life Impact
}

\author{
Jin Jiao, Rui Pang*, Yanling Li \\ Affiliated Hospital of Hebei University, Baoding 071000, Hebei Province, China \\ *Corresponding author: Rui Pang, pang1523128992@163.com
}

\begin{abstract}
Multiple studies have demonstrated that oral health quality of life (OHQOL) in patients with type 2 diabetes (T2DM) is affected by multiple factors. Therefore, during this study the related factors of OHQOL for T2DM was reviewed, and analyzed them from the aspects of socio-demographic factors, T2DM related factors and oral health related factors. This was to provide basis for nursing workers to develop personalized nursing measures.
\end{abstract}

Keywords: Type 2 diabetes; Oral health quality of life; Influencing factors; Review

Publication date: July 2021; Online publication: July 31, 2021

\section{Introduction}

Diabetes mellitus (DM) is divided into type 1 Diabetes mellitus, type 2 Diabetes mellitus, gestational Diabetes mellitus and other types of Diabetes mellitus, where T2DM is the majority in China. High levels of blood sugar in the body leads to a variety of complications, including oral complications. DM and periodontal disease interacts to form a vicious circle, but it hasn't gotten the clinical or patient attention it deserves ${ }^{[1]}$. As clinical medical staff, attention should be paid to the oral health status of patients with T2DM and to improve the oral health quality of life of patients with T2DM.

\section{Related concepts}

\subsection{Type 2 diabetes}

T2DM is characterized by progressive blood glucose elevation, and blood vessels and nerves are filled with high blood glucose for a long time, which is prone to various complications ${ }^{[2]}$. Oral cavity is the most vulnerable site to be affected by blood glucose, and most of them have the problem of xerostomia, among which the incidence of periodontal disease is the highest ${ }^{[3]}$.

\subsection{Oral health and quality of life}

Different researchers have proposed different concepts for oral health quality of life (see Table 1.).

\subsection{The relationship between diabetes mellitus and oral health quality of life}

The incidence of periodontal disease in DM patients is significantly higher than that in non-DM patients ${ }^{[4]}$. T2DM has a bidirectional relationship with OHQOL ${ }^{[5]}$, that is, T2DM can lead to an increased incidence of oral diseases, and poor oral health status leads to poor blood glucose control.

The mechanism of diabetes mellitus and oral health quality of life are related to the interaction of multiple systems:

(1) The effects of T2DM on OHQOL: The salivary gland was damaged; Oral infection; Plasma adiponectin levels changes, whereby DM leads to periodontal vascular damage and weakened 
mucosal repair, leading to more oral diseases ${ }^{[4]}$.

(2) Effects of OHQOL on T2DM: Low degree inflammation where, periodontitis leads to increased activity of inflammatory factors in the oral cavity, leading to decreased sensitivity to insulin, and aggravating insulin resistance ${ }^{[4]}$. For tooth loss, the existence of periodontitis leads to fragile tooth matrix, then masticatory dysfunction, followed by nutritional disorders, resulting in poor blood sugar control.

Table 1. The concept of oral health quality of life

\begin{tabular}{|c|c|c|}
\hline Years & Author & Concept \\
\hline 1993 & Dolan $\mathrm{T}$, et al & $\begin{array}{l}\text { Oral health is defined as a comfortable and well-functioning dentition } \\
\text { that enables individuals to continue to perform their desired social } \\
\text { roles. }\end{array}$ \\
\hline 1997 & Gift HC, et al & $\begin{array}{l}\text { These include survival (no presence of oral cancer); Free from } \\
\text { damage and disease; No discomfort or pain associated with chewing } \\
\text { or swallowing; Emotional functions related to smiling; Social } \\
\text { functions related to normal roles; Perception and satisfaction with oral } \\
\text { health; And lack of social or cultural disadvantage due to oral } \\
\text { conditions. }\end{array}$ \\
\hline 2000 & Locker D, et al & $\begin{array}{l}\text { Consideration needs to be given to the measurement of clinical } \\
\text { indicators of oral disease, the functional and psychosocial } \\
\text { implications of oral condition, and mental health and life satisfaction. }\end{array}$ \\
\hline 2011 & Inglehart MR, et al & $\begin{array}{l}\text { Consideration should be given to the individual's assessment of how } \\
\text { the following factors affect their health: functional factors, } \\
\text { psychological factors, social factors, and oral and facial } \\
\text { pain/discomfort experiences. }\end{array}$ \\
\hline
\end{tabular}

\section{Influencing factors of OHQOL in T2DM patients}

\subsection{Sociodemographic elements}

\subsubsection{Age}

Age increases the risk of periodontitis in DM patients ${ }^{[6]}$. However, some studies have found that the relationship between DM and tooth loss is closer in the younger group ( $\leq 65$ years old) than in the older group ( $\geq 65$ years old) ${ }^{[7]}$. Age is a factor affecting of OHQOL, but the tendency of different ages to suffer from oral diseases and the ways through which age factors affect oral health still need to be further studied.

\subsubsection{Gender}

The prevalence of moderate and severe periodontitis in males is higher than that in females ${ }^{[8]}$. However, some studies have found that although the rate of tooth loss in males is higher than in females, this correlation is not significant ${ }^{[9]}$. Therefore, gender is an influential factor of oral health quality of life in patients with T2DM, which still needs some evidence to support.

\subsubsection{Body Mass Index (BMI)}

Most patients with T2DM are obese. The mean gingival atrophy of obese people is higher than of nonobese people ${ }^{[10]}$. BMI in patients with T2DM is correlated with periodontitis and tooth loss ${ }^{[11,12]}$. In conclusion, BMI is an important risk factor for the deterioration of oral health quality of life in patients with T2DM. 


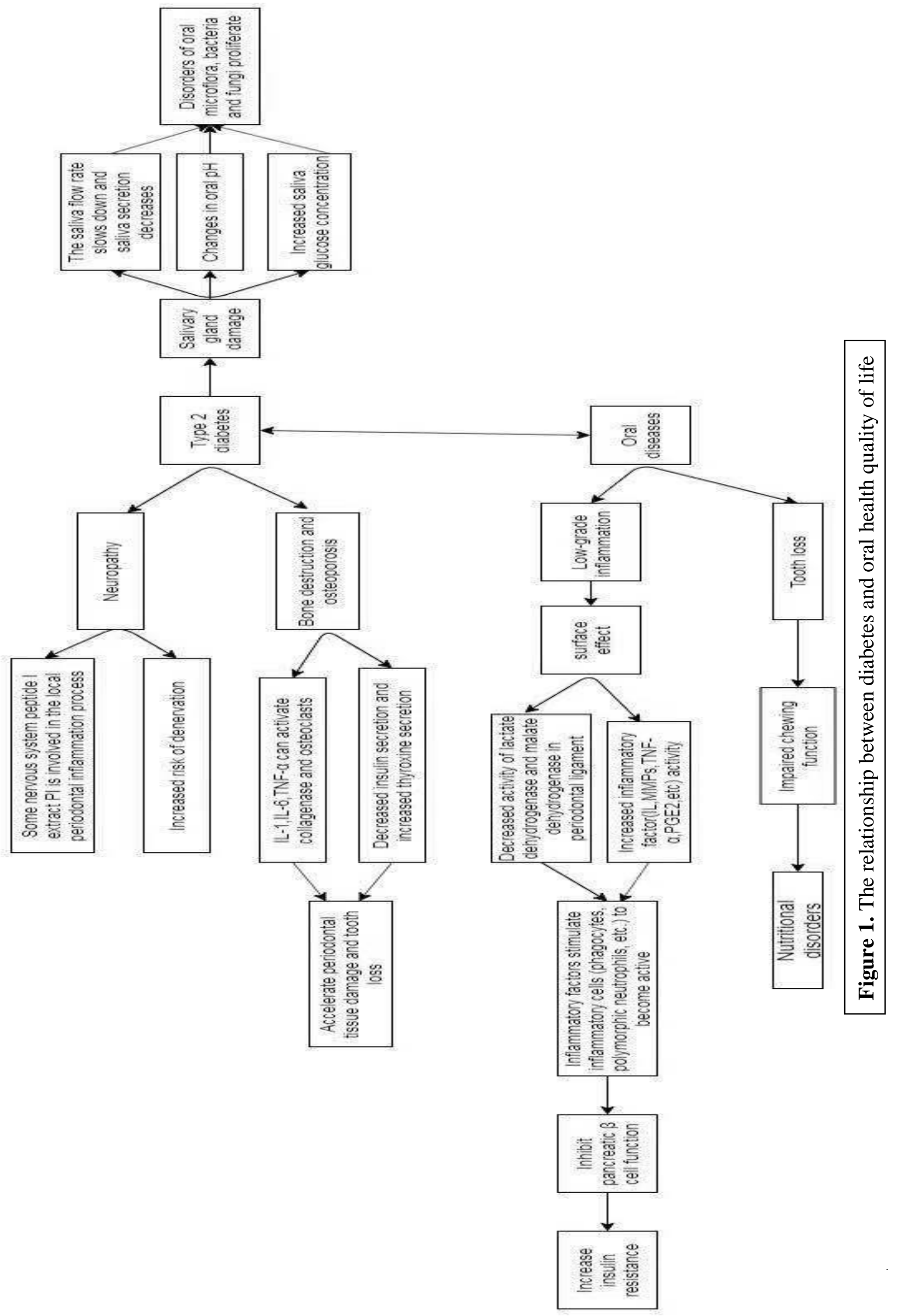




\subsubsection{Education level}

Studies have shown that education level is significantly correlated with the number of missing teeth ${ }^{[13]}$, and poor school education is an important factor for the occurrence of periodontal disease in patients with $\mathrm{T}^{\mathrm{DMM}}{ }^{[14]}$. People with higher education level have better oral health and life quality ${ }^{[15]}$.

\subsubsection{Income status}

Family income is one of the determinants affecting the oral health quality of patients with T2DM ${ }^{[9]}$. The higher the income, the better the oral health status ${ }^{[16]}$. It is necessary to strengthen the support for T2DM patients with poor economic income and improve their awareness and material basis of oral health care.

\subsubsection{Dental insurance}

Dental insurance plays an important role in the oral health of T2DM patients ${ }^{[17]}$. Dental insurance can protect the oral health of T2DM population and reduce their economic burden.

\section{2. $T 2 D M$ related factors}

\subsubsection{T2DM duration}

Some scholars have found that the duration of T2DM is significantly correlated with periodontal index ${ }^{[18]}$, while Han, $\mathrm{K}$ et al. ${ }^{[6]}$ did not find the relationship between periodontitis and the duration of T2DM. Different researchers hold different conclusions about the effect of the course of T2DM on oral health.

\subsubsection{T2DM self-management}

Studies have shown that patients with good blood glucose control have higher OHQOL than those with poor blood glucose control, and periodontal treatment is beneficial to blood glucose control in patients with $\mathrm{T} \mathrm{DM}^{[7]}$. Zhu Meihong et al. ${ }^{[19]}$ believed that OHQOL of DM patients with good treatment compliance was higher than those with poor compliance. Therefore, T2DM patients should pay attention to selfmanagement of controlling blood glucose level to improve OHQOL.

\subsection{Factors related to oral health}

\subsubsection{Oral health knowledge and attitude}

People with good knowledge of oral health have better OHQOL ${ }^{[20]}$. T2DM patients with positive attitudes towards periodontal health have better glycemic control ${ }^{[21]}$. In conclusion, oral health knowledge and attitude can affect the oral health quality of life of T2DM patients.

\subsubsection{Oral health behavior}

The study found that T2DM patients with periodontal treatment experience pay more attention to tooth cleaning ${ }^{[21]}$. The use of secondary oral products can reduce the incidence of periodontitis in T2DM ${ }^{[6]}$. Oral health behaviors of DM patients in China are generally poor ${ }^{[19,22]}$. Therefore, medical staff should guide DM patients to pay attention to and strengthen good oral health behaviors to improve OHQOL.

\subsubsection{Oral health resources}

Studies have shown that regular periodontal follow-up can improve oral health quality of life in $91 \%$ of patients ${ }^{[23]}$. Access to oral health resources for T2DM patients should be facilitated through various means.

\section{Conclusion}

In conclusion, oral health quality of life of patients with T2DM is affected by a variety of factors, mainly including socio-demographic factors, T2DM related factors and oral health related factors. Although the influencing factors of oral health quality of life include uncontrollable factors such as age and gender, we can improve the oral health quality of life of T2DM patients by changing the controllable factors. 


\section{Acknowledgments}

This work was supported by Hebei Provincial Administration of Traditional Chinese, and it was used in the research project of Chinese medicine in 2019, the item number: 2019178.

\section{Disclosure statement}

The author declares no conflict of interest.

This review article cites the peer-reviewed manuscripts of other groups. This review does not include a study design with the direct ethical statements of the human or animal study designs.

\section{Author contributions}

Li YL, Wang XJ, and Yan YQ conceptualized the questions and developed protocol for the review article. Zhang LL, Yu GF and Wang YL wrote writing-original draft preparation. Li YL critically reviewed and edited the manuscript. All authors have read and given final approval of the article to be published.

\section{References}

[1] Zhang J, Ding J, 2020, Study on The Periodontal Status and Influencing Factors of Type 2 Diabetes Patients with Family Doctor Contract in Beijing Yuetan Community. Chinese Journal of General Practice, 23(13): 1705-1710

[2] Zheng Y, Ley SH, Hu FB, 2018, Global Aetiology and Epidemiology of Type 2 Diabetes Mellitus and Its Complications. Nat Rev Endocrinol, 14(2): 88-98.

[3] Rohani B, 2019, Oral Manifestations in Patients with Diabetes Mellitus. World J Diabetes, 10(9): 485-489.

[4] Wernicke K, Zeissler S, Mooren FC, et al., 2018, Probing Depth is An Independent Risk Factor for HbA1c Levels in Diabetic Patients under Physical Training: A Cross-Sectional Pilot-Study. BMC Oral Health, 18(1): 46.

[5] Gurav AN, 2016, Management of Diabolical Diabetes Mellitus and Periodontitis Nexus: Are We Doing Enough?. World J Diabetes, 7(4): 50-66.

[6] Han K, Park JB, 2018, Clinical Implications of Age and Sex in the Prevalence of Periodontitis in Korean Adults with Diabetes. Exp Ther Med, 15(4): 3865-3873.

[7] Similä T, Auvinen J, Puukka K, et al., 2018, Impaired Glucose Metabolism is Associated with Tooth Loss in Middle-Aged Adults: The Northern Finland Birth Cohort Study 1966. Diabetes Research and Clinical Practice, 142:110-119.

[8] Liu Y, Yu Y, Nickel JC, et al., 2018, Gender Differences in the Association of Periodontitis and Type 2 Diabetes. Int Dent J, 68(6): 433-440.

[9] Hastings JF, Vasquez E, 2017, Diabetes and Tooth Loss among Working-Age African Americans: A National Perspective. Soc Work Public Health, 32(7): 443-451.

[10] Deshpande NC, Amrutiya MR, 2017, Obesity and Oral Health - Is There A Link? An Observational Study. J Indian Soc Periodontol, 21(3): 229-233.

[11] Pereira ALP, Frias AC, Hasegawa CCT, et al., 2018, Assessment Between Dental Caries Index and Body Mass Index among Adults. Oral Health Prev Dent, 16(6): 563-569.

[12] Khanuja PK, Narula SC, Rajput R, et al., 2017, Association of Periodontal Disease with Glycemic Control in Patients with Type 2 Diabetes in Indian Population. Front Med, 11(1): 110-119. 
[13] I Sebai, Temessek A, Chelly A, et al., 2019, Assessment of Oral Health Status Among Uncontrolled Diabetic Mellitus Patients in Tunisia. Tunis Med, 97(2): 307-313.

[14] F Javed, Tenenbaum H-C, Nogueira-Filho G, et al., 2013, Severity of Periodontal Disease in Individuals Chewing Betel Quid With and Without Tobacco. Am J Med Sci, 346(4): 273-278.

[15] M Alasqah, Mokeem S, Alrahlah A, et al., 2018, Periodontal Parameters in Prediabetes, Type 2 Diabetes Mellitus, and Non-Diabetic Patients. Braz Oral Res, 32:81.

[16] Nazer FW, Sabbah W, 2018, Do Socioeconomic Conditions Explain Ethnic Inequalities in Tooth Loss among US Adults?. Ethn Dis, 28(3): 201-206.

[17] N-M Marlow, Slate E-H, Fernandes J-K, et al., 2013, Associations between Health Insurance and Generalized Periodontal Disease in a Study Population of Gullah African Americans with Type-2 Diabetes. Community Dent Oral Epidemiol, 41(1): 40-47.

[18] E-K Kim, Lee S-G, Choi Y-H, et al., 2013, Association between Diabetes-Related Factors and Clinical Periodontal Parameters in Type-2 Diabetes Mellitus. BMC Oral Health, 13:64.

[19] Zhu Meihong, Wang Wenlan, Chen Qiongfang, et al., 2011, Oral Health Status of Diabetic Patients and its Influencing Factors. Journal of Nursing, 26(17): 54-56.

[20] Shimpi N, Glurich I, Schroeder D, et al., 2020, Patient Awareness of Association of Diabetes and Periodontal Disease. Health Promot Pract, 21(3): 464-472.

[21] Y-J Hsu, Lin K-D, Chen J-H, et al., 2019, Periodontal Treatment Experience Associated with Oral Health-Related Quality of Life in Patients with Poor Glycemic Control in Type 2 Diabetes: A Case-Control Study. Int J Environ Res Public Health, 16(20): 4011.

[22] Song H, Gao M, Liu W, 2016, Study on the Relationship between the Quality of Oral Health and the Degree of Oral Knowledge in Elderly Patients with Diabetes. Journal of Taishan Medical College, 37(8): 841-843.

[23] A Aljaber, Al-Surimi K, 2015, Promoting Oral Health Practice Among Patients with Diabetes Attending Primary Health Care Clinics. BMJ Qual Improv Rep, 4(1): 172. 